\title{
Frailty and Malnutrition in a cohort of Irish community-dwelling older adults
}

\author{
M.L. O'Connell ${ }^{1}$, T. Coppinger ${ }^{2}$ and A.L. McCarthy ${ }^{1}$ \\ ${ }^{1}$ Department of Biological Sciences, Munster Technological University, Rossa Avenue, Bishopstown, Cork, Republic of \\ Ireland and \\ ${ }^{2}$ Department of Sport, Leisure and Childhood Studies, Munster Technological University, Rossa Avenue, Bishopstown, \\ Cork, Republic of Ireland
}

Frailty and malnutrition are widespread syndromes in older adults, and are both linked to increased risk for dependency, hospitalisation and mortality ${ }^{(1)}$. Evidence suggests that these conditions share common causal factors and physiological pathways, such as body composition changes and loss of tissue mass ${ }^{(2)}$. The objectives of this study were to investigate the prevalence of, and association between, frailty and malnutrition, in addition to factors associated with each condition, in a cohort of Irish adults aged $\geq 65$ years.

A total of 142 community-dwelling older volunteers $(n=81$ female, $n=61$ male, $74.1 \pm 6.80$ years $)$ were recruited to participate through advertisement in community settings in Cork, Ireland (March-August, 2019). Community-based screening sessions were held for those eligible, where frailty, nutritional status and dietary variables were measured. Frailty was classified according to the Frailty Phenotype, described as $\geq 3$ (or 1-2 for pre-frailty) of the following features; weakness, exhaustion, low activity, slowness and weight loss. Nutritional status was assessed using the Mini Nutritional Assessment - Short Form (MNA-SF). Supplement use and intake of protein $(\% \mathrm{E})$ and alcohol $(\mathrm{g})$ were recorded using a validated Food Frequency Questionnaire (FFQ). Pearson's chi-squared test and logistic regression analysis were performed to investigate the association between frailty, malnutrition and demographic and dietary variables. Ethical approval was obtained from C.I.T. Research Ethics Committee, Cork, Ireland.

The prevalence of pre-frailty and frailty in this cohort were $34.5 \%$ and $12.0 \%$, respectively. Only one participant was malnourished, thus, those who were malnourished and at risk of malnutrition were grouped together for analysis, resulting in a prevalence of $19.7 \%$. Although not statistically significant ( $\mathrm{p}>0.05)$, compared to males, females were more likely to be malnourished/at risk $(22.0 \%$ vs $16.4 \%)$, and more likely to be pre-frail or frail $(50.6 \%$ vs $41.0 \%) .75 .0 \%$ of those at risk of malnutrition were also pre-frail or frail, compared to only $39.5 \%$ of those who were well-nourished $(\mathrm{p}<0.001)$. Use of dietary supplements decreased the risk for malnutrition [odds ratio $(\mathrm{OR})(95 \% \mathrm{CI}): 0.19(0.04-0.68), \mathrm{p}=0.021$ ], while being pre-frail or frail (compared to non-frail) increased the risk for malnutrition [OR: $5.06(1.82-15.56), \mathrm{p}=0.003$ ]. Increasing age, being malnourished/at risk (compared to well-nourished) and having a low protein intake were associated with an increased frailty risk [OR: $1.16(1.10-1.23), 5.83$ (2.36-14.95) and 2.93 (1.16-7.62), respectively, all $\mathrm{p}<0.05]$

Our study revealed a frailty prevalence of $12.0 \%$ in this cohort, while $19.7 \%$ were malnourished or at risk of malnutrition. Despite a divergence in risk factors, and a proven ability to exist independently, a significant overlap exists between frailty and malnutrition. The direction of this association remains unclear, however, and warrants further exploration. Nonetheless, nutritional considerations are important for the development of strategies to reduce/prevent frailty.

\section{References}

1. Hébert R (2017) JAMDA 18, 1019-28.

2. Jeejeebhoy KN (2012) Curr Opin Clin Nutr Metab Care 15, 213-19. 